\title{
Comparison between glycerol and ethylene glycol for the cryopreservation of equine spermatozoa: semen quality assessment with standard analyses and with the hypoosmotic swelling test
}

\author{
Roberto MANTOVANI $^{\mathrm{a} *}$, Ada Rota ${ }^{\mathrm{b}}$, Maria Elena FALOMO ${ }^{\mathrm{b}}$, \\ Lucia BAILONI $^{\mathrm{a}}$, Leila VINCENTI ${ }^{\mathrm{c}}$ \\ ${ }^{a}$ Department of Animal Science, University of Padua, Agripolis, 35020 Legnaro (PD), Italy \\ ${ }^{b}$ Department of Veterinary Clinical Sciences, University of Padua, Italy \\ ${ }^{c}$ Department of Animal Pathology, Faculty of Veterinary Medicine, University of Turin, Italy
}

(Received 4 February 2002; accepted 21 May 2002)

\begin{abstract}
The aims of this study were to compare glycerol (G) at customary concentrations and ethylene glycol (EG) as cryoprotectants for stallion semen in a skimmed milk (SM) extender, to test different EG concentrations and to compare the results of manual and computerized analysis with the hypoosmotic swelling (HOS) test. Ejaculates from two stallions were collected over 3 weeks ( 6 ejaculates per stallion), diluted in a SM based extender, divided into 4 fractions, centrifuged and diluted again to a concentration of $100 \times 10^{6} \mathrm{~mL}^{-1}$ progressive motile spermatozoa (PMS) in addition with the cryoprotectant (3\% G, 3\% EG, 6\% EG, 9\% EG). Sperm motility was assessed both by microscopy (in raw and frozen-thawed semen immediately after thawing) and with an HTM-IVOS analyzer (Hamilton-Thorne Research, MA, USA), at $0,1,4,6$, and $12 \mathrm{~h}$ after thawing and storage at $21^{\circ} \mathrm{C}$. Raw and frozen-thawed $(0 \mathrm{~h})$ semen samples for $\mathrm{G}$ and $\mathrm{EG}$ at $3 \%$ were also submitted to the HOS test with a $100 \mathrm{mOsm}$ sucrose solution and were evaluated to detect the presence of swollen tails. The higher EG concentrations (i.e. 6\% EG and 9\% EG) significantly reduced the percentage of motile and PMS, immediately after thawing. At the same concentration, i.e. 3\%, G resulted in a higher percentage of PMS than EG (36.2 vs. 30\%, $P<0.05)$, but at $12 \mathrm{~h}$ after thawing and storage at $21^{\circ} \mathrm{C}$, no significant differences were detected between $\mathrm{G}$ and EG at $3 \%$. The correlations between progressive motility (assessed by direct microscope observation or measured through the HTM analyzer) and the HOS test results for $3 \% \mathrm{G}$ and EG were $r=0.61$ and $r=0.35$, respectively. The HOS test confirmed its suitability as a complementary method of analysis for stallion semen. We conclude that with the SM extender used, EG could substitute G as the cryoprotectant for stallion semen if used at the same or lower concentration.
\end{abstract}

stallion / semen / cryoprotectant / glycerol / ethylene glycol

* Correspondence and reprints

E-mail: roberto.mantovani@unipd.it 


\section{INTRODUCTION}

Many extenders in various combinations with cryoprotectants are used for processing equine semen, but the large variability in post-thawing sperm survival and in field fertility among stallions indicates that many factors can still be improved [4].

Glycerol was the first cryoprotectant used for freezing sperm and it is actually the most largely employed for equine semen [9, 29] although it is believed to be involved in poor post-thaw motility [19]. Other compounds have been tested only sporadically in the past; good results were obtained with dimethyl sulfoxide (DMSO) [7] and also with EG [2]. EG has a low molecular weight (62.07), lower than G (92.10) or DMSO (78.13), and this feature, together with its high permeability, makes it suitable as a cryoprotectant for embryo freezing or vitrification procedures, whereas its low toxicity makes a stepwise dilution unnecessary [16].

The parameters routinely measured to express semen quality are concentration, motility, and morphology. A test detecting sperm membrane functionality, like the hypoosmotic swelling (HOS) test, could easily be included in standard semen analyses. The HOS test consists in challenging spermatozoa with a hypoosmotic solution: if the plasma-membrane is functionally active it can modulate water permeability and the resultant osmotic equilibrium produces a swelling and coiling of the sperm tail. No swelling occurs if the sperm membrane is damaged. The HOS test has been adopted to evaluate sperm quality in several species, including man $[11,14,28]$, cattle $[8,23$, 24], and sheep [22], and it has also been experimented in horses $[6,17,18]$.

The objectives of this study were to compare glycerol $(\mathrm{G})$ and ethylene glycol (EG) at different concentrations as cryoprotectants for stallion semen and to compare standard semen parameters with the HOS test results, in fresh and frozen-thawed semen.

\section{MATERIALS AND METHODS}

\subsection{Semen collection and processing}

Six ejaculates from two mature stallions of proven fertility and in good body condition were collected with a Missouri model artificial vagina.

The gel fraction was removed by filtration. Semen was kept at $37{ }^{\circ} \mathrm{C}$ and it was diluted 1:4 with a Skimmed Milk Extender [21] pre-warmed at $37^{\circ} \mathrm{C}$. After dilution, the ejaculates were split into four aliquots and centrifuged at $350 \times g$ at $4{ }^{\circ} \mathrm{C}$ for $25 \mathrm{~min}$ (cooling rate of approximately $1.3{ }^{\circ} \mathrm{C} \mathrm{min}-1$ ). The supernatant was discarded and the sperm pellet was re-suspended in $10 \mathrm{~mL}$ of the previous extender (cooled at $4{ }^{\circ} \mathrm{C}$ ), supplemented with $4 \%$ egg yolk and one of the tested cryoprotectants ( $\mathrm{G}$ and $\mathrm{EG}$ ), to obtain the following final concentrations: $3 \% \mathrm{G}$, $3 \% \mathrm{EG}, 6 \% \mathrm{EG}$, and $9 \%$ EG. A standardized sperm concentration was reached adjusting at $100 \times 10^{6}$ progressively motile spermatozoa $\cdot \mathrm{mL}^{-1}$. All the samples were kept in a water bath at $4{ }^{\circ} \mathrm{C}$ for $1 \mathrm{~h}$ and then loaded into $0.5 \mathrm{~mL}$ straws. After packaging, semen temperature increased to $6{ }^{\circ} \mathrm{C}$. The straws were then introduced into the freezing chamber of a programmable freezer, and held at $4{ }^{\circ} \mathrm{C}$ for $3 \mathrm{~min}$ to stabilize the temperature again before freezing. Freezing took place at a cooling rate of $60{ }^{\circ} \mathrm{C} \mathrm{min}-1$ until $-140{ }^{\circ} \mathrm{C}$, followed by plunging the straws into liquid nitrogen. Straws were thawed at $37{ }^{\circ} \mathrm{C}$ for $30 \mathrm{~s}$.

\subsection{Semen quality evaluation}

Sperm was evaluated after collection, after the first and the second extension (the latter with the cryoprotectant) and then after freezing/thawing: progressive motility (PM\%) was directly assessed by microscope observation, on a $4 \mu \mathrm{L}$ drop of semen placed on a pre-warmed gridded glass slide (Cell-Vu ${ }^{\circledR}$ chamber - $1 \mathrm{~mm}$ depth) on a warmed stage $\left(37^{\circ} \mathrm{C}\right)$. 
Thawed sperm was also analyzed with an HTM-IVOS sperm motility analyzer (Hamilton-Thorne Research, MA, USA; Version 10.6t). Samples were kept at $21^{\circ} \mathrm{C}$ and were evaluated at different times, i.e. immediately after thawing (time 0 ) and then after $1,4,6$, and $12 \mathrm{~h}$. The settings for the HTM, drawn from previous works [12, 20], were as follows: frame acquired, 20 (min no. 15 ); acquisition rate $50 \mathrm{~Hz}$; min contrast, 45; min cell size, 4; threshold straightness, 80; medium velocity (VEL) cut off, $25 \mu \mathrm{m} \cdot \mathrm{s}^{-1}$; low VEL cut off, $24.9 \mu \mathrm{m} \cdot \mathrm{s}^{-1}$, non motile head size, 13 ; non motile head intensity, 25; static size limits, 0.53-3.40; static intensity limits, 0.26-3.36; static elongation limits, 12-80. A preliminary measure of repeatability within the sample was assessed and, analyses started when motility parameters differed $\leq 3 \%$ between subsequent measurement. Two straws were mixed and analyzed for each sample: a $4 \mu \mathrm{L}$ aliquot of semen was diluted 1:1 with the prewarmed skimmed milk extender [21], then it was placed on a warmed Cell-Vu ${ }^{\circledR}$ chamber and five fields per chamber were analyzed. The measured motion parameters were motility (M\%), progressive motility (PM\%), path velocity (VAP, $\mu \mathrm{m} \cdot \mathrm{s}^{-1}$ ), track speed (VCL, $\mu \mathrm{m} \cdot \mathrm{s}^{-1}$ ), progressive velocity $\left(\mathrm{VSL}, \mu \mathrm{m} \cdot \mathrm{s}^{-1}\right)$, straightness (STR = VSL/VAP), and linearity (LIN = VSL/VCL).

Samples of raw, extended with the cryoprotectants ( $3 \%$ concentration of $\mathrm{G}$ and EG), and frozen/thawed semen (at time 0) were subjected to the HOS test. Aliquots of semen of $50 \mu \mathrm{L}$ were incubated in $500 \mu \mathrm{L}$ of a hypoosmotic solution, i.e. a sucrose solution with an osmolarity of 100 mOsm [18]. The addition of semen increased the osmolarity to approximately $150 \mathrm{mOsm}$ with both the cryoprotectants. After $10 \mathrm{~min}$ incubation at $25^{\circ} \mathrm{C}$, at least 100 spermatozoa per sample were observed under light microscopy $(400 \times)$, and were then scored according to the presence or not of swollen tails.

\subsection{Statistical analyses}

The data measured immediately after thawing using both optic microscopy and image analysis methods were subjected to ANOVA, using a linear model for a splitplot design, with the collection date and the stallion effects in the main plot, and the cryoprotectant effect (3\% G, 3\% EG, 6\% EG, $9 \%$ EG) and the interaction between the cryoprotectant and stallion in the sub-plot. The following orthogonal contrasts were tested for the cryoprotectant effect: $3 \% \mathrm{G}$ versus $3 \% \mathrm{EG}$, linear and quadratic components for increasing EG concentrations.

The same model was adopted for the analysis of data obtained with the HOS test, comparing $\mathrm{G}$ and $\mathrm{EG}$ at the same $3 \%$ concentration.

Data recorded for motility and progressive motility at thawing and at different postthawing times $(1,4,6$, and $12 \mathrm{~h})$ were subjected to a preliminary analysis in order to describe a decay curve, i.e. the variation of semen characteristics over time. The exponential model as $y=b \cdot e^{(-c t)}$ was fitted by a non-linear procedure and the DUDE method [26]. The values of $b$ ( $y$ axis intercept or estimated value of the parameter at time $\mathrm{t}=0$ ) and c (rate of decay, $\% \cdot \mathrm{h}^{-1}$ ) for M\% and PM\% were then analyzed by ANOVA as previously described.

The correlation coefficients between the HOS test results and progressive motility (obtained both by direct microscope observation and by the HTM assessments) were calculated in frozen-thawed semen.

\section{RESULTS}

Table I shows semen quality parameters (mean values from six ejaculates) before freezing. The values observed were very similar for the two animals and the results of the HOS test show a lower variability than the evaluation of PM\%. 
Table I. Percentage of progressively motile spermatozoa (microscope analysis) and of spermatozoa showing swollen tails, in raw and extended semen before freezing (mean values \pm standard deviation)

\begin{tabular}{lcc}
\hline & PM\% & HOS $+\%$ \\
\hline Stallion 1: & & \\
$\quad$ Raw semen & $84.8 \pm 8.3$ & $92.5 \pm 1.6$ \\
Plus cryoprotectant: & $83.7 \pm 3.4$ & $89.5 \pm 1.4$ \\
3\% glycerol & $84.5 \pm 4.4$ & $89.5 \pm 1.9$ \\
3\% ethylene glycol & $75.7 \pm 6.8$ & - \\
6\% ethylene glycol & $65.6 \pm 10.3$ & - \\
9\% ethylene glycol & & \\
Stallion 2: & $71.8 \pm 11.8$ & $91.0 \pm 2.2$ \\
Raw semen & & $89.5 \pm 4.3$ \\
Plus cryoprotectant: & $80.3 \pm 5.8$ & $88.2 \pm 1.9$ \\
3\% glycerol & $84.5 \pm 4.7$ & - \\
3\% ethylene glycol & $80.2 \pm 6.7$ & - \\
6\% ethylene glycol & $69.0 \pm 16.7$ & \\
9\% ethylene glycol & & \\
\hline
\end{tabular}

$\mathrm{PM} \%=$ progressive motility $(\%)$.

$\mathrm{HOS}+\%=$ percentage of spermatozoa showing swollen tails.

ANOVA indicated no significant interaction between the cryoprotectant and the stallion; thus, only the results regarding the cryoprotectant effect are presented.

The comparisons between mean values obtained by direct microscope observation, by HTM analysis and by the HOS test immediately after thawing appear in Table II. The cryoprotectant concentration $(3 \% \mathrm{G}, 3 \%$ $\mathrm{EG}, 6 \% \mathrm{EG}$, and $9 \% \mathrm{EG}$ ) had a significant effect on semen characteristics, as indicated by optic microscopy and HTM analysis for the main level. When the same concentration (3\%) of $\mathrm{G}$ and EG was compared, differences in PM\% were statistically significant $(P<0.05)$ only when the value came from the HTM analysis $(36.2 \%$ versus $30.0 \%$, respectively). The correlation coefficient between PM\% resulting from visual estimation and HTM reading was $53.5 \%$ $(P<0.01 ; n=48$, i.e. 2 stallions $\times 6$ ejaculates $\times 4$ cryoprotectants). The HOS test results were not different between $3 \% \mathrm{G}$ and $3 \% \mathrm{EG}$, either.
An increase in EG concentration caused a significant $(P<0.01)$ decrease in semen quality, following a linear pattern, while the quadratic effect was never significant.

The correlations between the progressive motility and the HOS test results were $r=0.61(P<0.01)$ and $r=0.35(P=0.09)$ for microscopy and HTM assessments, respectively $(n=24)$.

As for the other motility parameters, the only significant difference was related to straightness, which was higher with $3 \% \mathrm{G}$ than with $3 \%$ EG $(P<0.05)$.

The cryoprotectant significantly affected the " $b$ " values of the decay curves of motility and progressive motility, estimated up to twelve hours after thawing (Figs. 1 and 2 respectively). An increase in EG concentration always produced a linear decrease in $\mathrm{M} \%$ and PM\% (linear contrast $P<0.01$ ).

The rate of decay ("c") was not affected by the substitution of the cryoprotectant $(\mathrm{G}$ vs. EG) or by an increasing EG concentration, 
Table II. Quality parameters of thawed semen (time 0) measured with optic microscopy (Microscope), with Hamilton (HTM) or with the HOS test.

\begin{tabular}{|c|c|c|c|c|c|c|c|c|}
\hline & \multirow{3}{*}{$\begin{array}{c}\text { Glycerol } \\
\text { (G) } \\
3 \%\end{array}$} & \multirow{2}{*}{\multicolumn{3}{|c|}{$\begin{array}{l}\text { Ethylene glycol } \\
\text { (EG) }\end{array}$}} & \multirow{3}{*}{$\mathrm{P}$} & \multicolumn{3}{|c|}{ Contrasts } \\
\hline & & & & & & \multirow{2}{*}{$\begin{array}{c}\text { G vs. EG } \\
(3 \%)\end{array}$} & \multirow{2}{*}{$\begin{array}{l}\text { EG } \\
\text { Lin. }\end{array}$} & \multirow{2}{*}{$\begin{array}{l}\text { EG } \\
\text { Quad. }\end{array}$} \\
\hline & & $3 \%$ & $6 \%$ & $9 \%$ & & & & \\
\hline \multicolumn{9}{|l|}{ Microscope } \\
\hline PM\% & 47.9 & 44.3 & 29.3 & 25.6 & $<0.001$ & NS & $<0.001$ & NS \\
\hline \multicolumn{9}{|l|}{ HTM } \\
\hline $\mathrm{M} \%$ & 64.9 & 58.8 & 44.9 & 29.9 & $<0.001$ & NS & $<0.001$ & NS \\
\hline PM\% & 36.2 & 30.0 & 19.3 & 12.3 & $<0.001$ & $<0.05$ & $<0.001$ & NS \\
\hline VAP & 78.7 & 78.8 & 77.1 & 68.3 & $<0.001$ & NS & $<0.001$ & NS \\
\hline VCL & 149.3 & 150.2 & 144.1 & 129.3 & $<0.001$ & NS & $<0.001$ & NS \\
\hline VSL & 61.7 & 60.4 & 56.6 & 48.6 & $<0.001$ & NS & $<0.001$ & NS \\
\hline STR & 75.0 & 72.9 & 69.1 & 67.1 & $<0.001$ & $<0.05$ & $<0.001$ & NS \\
\hline LIN & 40.7 & 39.6 & 38.5 & 37.6 & $<0.01$ & NS & $<0.01$ & NS \\
\hline \multicolumn{9}{|l|}{ HOS test } \\
\hline $\mathrm{HOS}+\%$ & 52.8 & 50.9 & - & - & NS & - & - & - \\
\hline
\end{tabular}

PM\% = progressive motility $(\%)$.

$\mathrm{M} \%=$ motility (\%).

$\mathrm{VAP}=$ path velocity $\left(\mu \mathrm{m} \cdot \mathrm{s}^{-1}\right)$

$\mathrm{VCL}=$ track speed $\left(\mu \mathrm{m} \cdot \mathrm{s}^{-1}\right)$.

VSL $=$ progressive velocity $\left(\mu \mathrm{m} \cdot \mathrm{s}^{-1}\right)$.

STR $=$ straightness $(\mathrm{VSL} / \mathrm{VAP})$.

LIN = linearity $(\mathrm{VSL} / \mathrm{VCL})$

$\mathrm{HOS}+\%=$ percentage of spermatozoa showing a swollen tail.

therefore the overtime decreases in $\mathrm{M} \%$ and PM\% showed a similar pattern.

The decay curves of PM\% with the same cryoprotectant concentration (3\%) had different $b$ values $(34.6 \%$ and $29.1 \%$ for $\mathrm{G}$ and $\mathrm{EG}$, respectively; $P<0.05)$; however, progressive motility showed similar values after $12 \mathrm{~h}$.
One hour after thawing, velocity parameters read by the HTM analyzer with $3 \% \mathrm{G}$ and $3 \%$ EG were not statistically different (Tab. III), whereas at the following readings some values were different; however, according to this comparison, the higher values of $\mathrm{G}$ appeared to be only transient.
Figure 1. Decay curve for motility (M\%) by experimental groups $(\mathrm{G}=$ glycerol; $\mathrm{EG}=$ ethylene glycol).

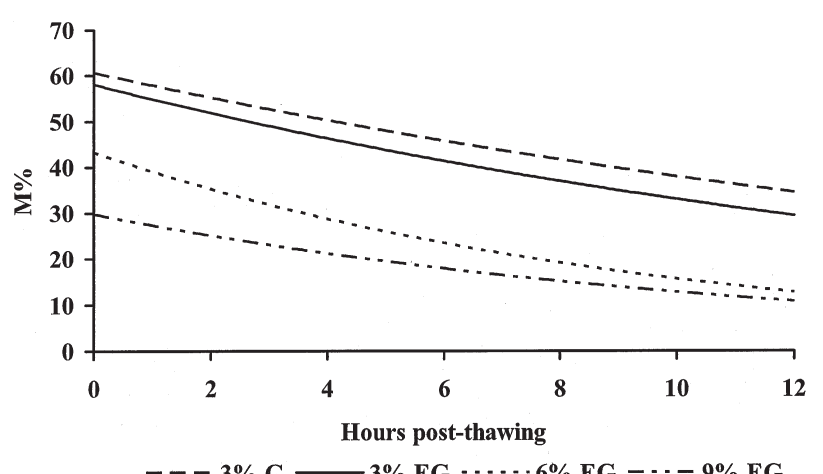

$---3 \%$ G $-3 \%$ EG $\cdots \cdots 6 \%$ EG $-\cdots-9 \%$ EG 


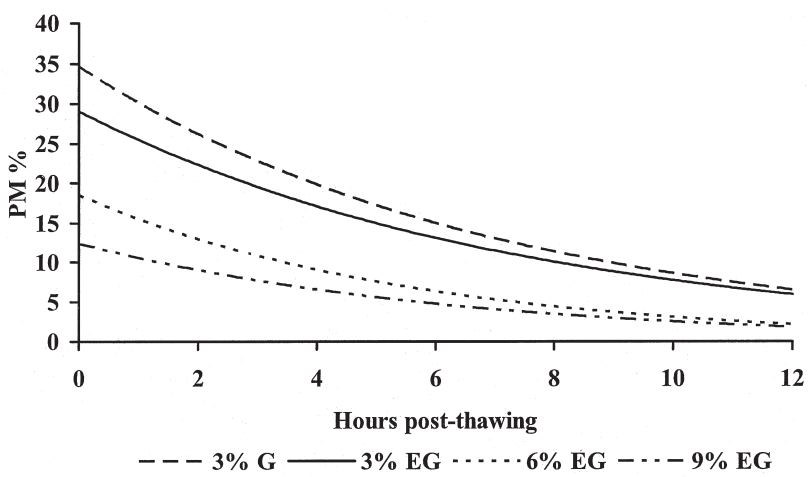

Figure 2. Decay curve for progressive motility (PM\%) by experimental groups $(\mathrm{G}=$ glycerol; $\mathrm{EG}=$ ethylene glycol).

Table III. Velocity parameters (HTM reading) with the same concentration of Glycerol and Ethylene glycol, respectively $1,4,6$, and $12 \mathrm{~h}$ post-thawing.

\begin{tabular}{lcc}
\hline & Glycerol 3\% & Ethylene glycol 3\% \\
\hline 1st h post-thaw & & \\
VAP & 77.9 & 81.3 \\
VCL & 151.3 & 156.3 \\
VSL & 59.6 & 60.7 \\
STR & 73.8 & 72.2 \\
LIN & 39.5 & 39.3 \\
4th h post-thaw & & \\
VAP & 85.5 & 84.2 \\
VCL & 167.0 & 165.1 \\
VSL & 61.3 & 58.2 \\
STR & $69.6^{\mathrm{a}}$ & $67.0^{\mathrm{b}}$ \\
LIN & 36.8 & 35.5 \\
6th h post-thaw & & \\
VAP & 91.1 & 87.5 \\
VCL & $179.2^{\mathrm{a}}$ & $169.5^{\mathrm{b}}$ \\
VSL & $62.1^{\mathrm{a}}$ & $58.3^{\mathrm{b}}$ \\
STR & $67.0^{\mathrm{A}}$ & $64.8^{\mathrm{B}}$ \\
LIN & 35.2 & 34.4 \\
12th post-thaw & & \\
VAP & 83.4 & 89.9 \\
VCL & 163.6 & 175.9 \\
VSL & 55.0 & 55.7 \\
STR & $64.4^{\mathrm{A}}$ & $61.3^{\mathrm{B}}$ \\
LIN & $34.7^{\mathrm{a}}$ & $32.6^{\mathrm{b}}$ \\
\hline
\end{tabular}

a, b Means differences at $P<0.05$ between columns.

A, B Means differences at $P<0.01$ between columns.

$\mathrm{VAP}=$ path velocity $\left(\mu \mathrm{m} \cdot \mathrm{s}^{-1}\right)$

$\mathrm{VCL}=$ track speed $\left(\mu \mathrm{m} \cdot \mathrm{s}^{-1}\right)$.

VSL $=$ progressive velocity $\left(\mu \mathrm{m} \cdot \mathrm{s}^{-1}\right)$.

STR $=$ straightness $($ VSL/VAP).

LIN = linearity (VSL/VCL). 


\section{DISCUSSION}

The methods to cryopreserve stallion semen vary largely between laboratories, currently lacking standardization in the various steps of the procedure [25]. In our work we followed the collection, freezing and thawing procedure currently adopted in a commercial laboratory.

Many factors besides the cryoprotectant affect post-thawing sperm survival, e.g. the cooling and warming rates or the different packaging techniques. Also spermatozoa in-straw concentration influences post-thawing semen motility parameters, with a decrease in both $\mathrm{M} \%$ and $\mathrm{PM} \%$ when sperm concentration is equal or exceeds $800 \times$ $10^{6}$ spermatozoa $\cdot \mathrm{mL}^{-1}[10]$. However, in a field trial [15] a very high spermatozoa concentration $\left(1600 \times 10^{6}\right.$ spermatozoa $\left.\cdot \mathrm{mL}^{-1}\right)$ resulted in a higher pregnancy rate. Since in our work we adjusted sperm concentration to the number of progressively motile spermatozoa, the total number of spermatozoa may have differed among the straws: however, the overall low variability in PM\% assures that the range of variation in sperm number per straw was limited and should not have affected sperm survival.

Glycerol is the cryoprotective agent used for equine semen in the majority of cases, at concentrations varying between 2.5 and $9 \%$ [25]. We know of only a few previous works in which EG was employed: Alvarenga et al. [3] showed a mean post-thawing motility of $36.5 \%$ with $5 \% \mathrm{EG}$, similar to $5 \% \mathrm{G}$ $(34.2 \%)$, while $10 \%$ EG caused a significantly smaller percentage of post-thaw motile spermatozoa (29.25\%); moreover, no difference was found in the number of spermatozoa with damaged acrosome or in the type of damage. However, an EDTAlactose-egg yolk extender was used in this study. On the contrary, the work by Chenier et al. [7], who used a milk-yolk extender, found a depression in post-thaw progressive motility due to EG (from 3 to $12 \%$ ) when compared to DMSO. Another study
[27] comparing $2.5 \%, 3.5 \%$ and $5 \% \mathrm{G}$ and EG, with an EDTA-glucose-egg yolk extender, showed significantly lower total and progressive motility with $2.5 \%$ EG only.

According to our data, $3 \%$ EG can be considered as the maximum level suitable to protect stallion sperm from cryo-damage, while higher concentrations show detrimental effects on semen quality. This level was lower than the concentration previously indicated by Alvarenga et al. [3] and Snoeck et al. [27], but the difference could partially be due to the different extenders which were used. It is indeed well-known that the extender significantly affects semen quality [4], therefore an interaction between the extender and cryoprotectant can be hypothesized, with the extender modulating water permeability occurring countercurrently to the cryoprotectant. Since in our study, EG concentrations higher than $3 \%$ negatively influenced semen quality, it could be interesting to test the efficacy (regarding semen protection and post-thawing quality preservation) of lower EG concentrations used together with the same extender we employed. Although we did not investigate on the supposed cryoprotectant toxicity, other Authors [3] failed to demonstrate that the cellular damage they observed was due to EG. In agreement with the same authors, we also noted that data concerning EG toxicity to spermatozoa are lacking. On the contrary, in a study characterizing the effects of addition and removal of different cryoprotectants for equine spermatozoa (1M G, EG, DMSO and Propylene Glycol) [5], EG showed the least detrimental effect on sperm viability and motility, while $\mathrm{G}$ caused the highest osmotic stress. Equine spermatozoa revealed a limited osmotic tolerance and GE was suggested as a valid cryoprotectant to reduce damages due to osmotic variations. In a recent study [1], another cryoprotectant showed a good potential for cryopreserving stallion semen, Dimethylformamide, both for motility parameters and for maintaining the ability of spermatozoa to bind to the chicken perivitelline membrane. 
Sperm membrane integrity can be evaluated with different assays, such as supravital staining or fluorescent labeling, but the HOS test, besides being simpler and more easily performed, also evaluates sperm membrane ability to modulate water and molecules passage [17]. In man, the HOS test is often routinely performed exactly because it has been proven to be a reliable way of assessing sperm functionality and detecting membrane integrity. Previous works have shown that raw and frozenthawed equine spermatozoa do react to the HOS test [17]. In our work we chose a $100 \mathrm{mOsm}$ sucrose solution which had given the maximum sperm swelling in previous research [18], but we shortened the incubation time to $10 \mathrm{~min}$, after a preliminary analysis of comparable efficacy (data not shown). We performed the test only with the same (3\%) cryoprotectants concentration since with the higher EG levels the final osmolarity was incomparably higher. In frozen-thawed bovine semen a good correlation was found between the percentage of motile spermatozoa and spermatozoa reacting to the HOS test [8]; in the stallion, the correlation between progressive motility and spermatozoa showing swelling was high $(r=0.75)$ in raw semen and lower $(r=0.57)$ in frozen-thawed semen, since progressive motility showed a greater susceptibility to cold shock than membrane functionality did [17]. These results are in contrast with our observations, since we found a correlation of $r=0.41$ between PM\% and HOS test results in raw semen and a higher correlation of $r=0.61$ in frozen-thawed semen.

In our work, the post-thawing percentage of spermatozoa with swollen tails was always higher than the percentage of progressively motile spermatozoa detected both by microscope analysis and with the HTM analyzer. On the contrary, when average motility instead of progressive motility was considered, its value was higher than the percentage of spermatozoa reacting to the HOS test (Tab. II). This was in agreement with previous works on bovine frozenthawed semen $[8,22]$, meaning that average motility may be over-estimated if coming from direct observation $[8,12]$. In our work, also the HTM analysis gave an overestimation of average motility; however, the setting of the "low velocity cut off" of the instrument that we used was $24.9 \mu \mathrm{m} \cdot \mathrm{s}^{-1}$, therefore a more accurate result might be obtained by refining this value.

Our work suggests that progressive motility of stallion semen can be accurately assessed by microscope analysis and that there is a good accordance between the different quality parameters we measured.

It remains to be investigated to what extent semen quality expressed through the laboratory analyses is an index of semen fertilizing ability. Unfortunately, it is known that semen quality measured through standard laboratory tests may be poorly correlated with the actual field fertility; particularly, in the stallion, the correlation between both visual and computer assisted estimation of sperm motility and fertility is rather poor $[9,13]$. In our work, the HOS test confirmed its potentiality as a useful complementary method of analysis for stallion semen; other, more complex investigations of actual semen fertilizing ability, such as sperm-oocyte interaction tests, could be taken into account to try to improve the correlation between field fertility and laboratory parameters.

In conclusion, our work showed the efficacy of EG as an alternative to the more common $\mathrm{G}$, as a cryoprotectant for stallion semen, when used at the same $(3 \%)$ or presumably lower concentration, in conjunction with a milk-egg yolk extender [21]. Despite the absence of field results and the low number of stallions we tested, our results could be useful for freezing semen of stallions which show low tolerability to Glycerol. 


\section{ACKNOWLEDGEMENTS}

The authors express their appreciation to Dr. P.R. Avezzù, Head of INTERMIZOO ${ }^{\circledR}$ Stud Center, where part of the work was done, for his indispensable cooperation. They are also grateful to the anonymous reviewers, whose suggestions greatly improved the manuscript.

\section{REFERENCES}

[1] Alvarenga M.A., Graham J.K., Keith S.L., Landim-Alvarenga F.C., Squires E.L., Alternative cryoprotectors for freezing stallion spermatozoa, Proc. XIV International Congress on Animal Reproduction (Stockholm), 2000, p. 157.

[2] Alvarenga M.A., Landim-Alvarenga F.C., Moreira R.M., Cesarino M.M., Utilization of ethylene glycol as a cryoprotector for equine semen, Proc. Soc. Theriogenology, Baltimore (MA), 1998, p. 155.

[3] Alvarenga M.A., Landim-Alvarenga F.C., Moreira R.M., Cesarino M.M., Acrosomal ultrastructure of stallion spermatozoa cryopreserved with ethylene glycol using two packaging systems, Equine Vet. J. 32 (2000) 541-545.

[4] Amann R.P., Pickett B.W., Principles of cryopreservation and a review of cryopreservation of stallion spermatozoa, J. Equine Vet. Sci. 7 (1987) 145-173.

[5] Ball B.A., Vo A., Osmotic tolerance of equine spermatozoa and the effects of soluble cryoprotectants on equine sperm motility, viability, and mitochondrial membrane potential, J. Androl. 22 (2001) 1061-1069.

[6] Caiza de la Cueva F.I., Rigau T., Bonet S., Mirò J., Briz M., Rodrìguez-Gil J.E., Subjecting horse spermatozoa to hypoosmotic incubation: effects of ouabain, Theriogenology 47 (1997) 765-784.

[7] Chenier T., Merkies K., Leibo S., Plante C., Johnson W., Evaluation of cryoprotective agents for use in the cryopreservation of equine spermatozoa, Proc. Soc. Theriogenology, Baltimore (MA), 1998, pp. 52-53.

[8] Correa J.R., Zavos P.M., The hypoosmotic swelling test: its employment as an assay to evaluate the functional integrity of the frozenthawed bovine sperm membrane, Theriogenology 42 (1994) 351-360.

[9] Graham J.K., Analysis of stallion semen and its relation to fertility, Vet. Clinics North America Equine Practice 12 (1996) 119-130.

[10] Heitland A.V., Jasko D.J., Squires E.L., Graham J.K., Pickett B.W., Hamilton C., Factors affecting motion characteristics of frozen-thawed stallion spermatozoa, Equine Vet. J. 28 (1996) 47-53.
[11] Hossain A.M., Rizk B., Barik S., Huff C. Thorneycroft I.H., Time course of hypo-osmotic swellings of human spermatozoa: evidence of ordered transition between swelling subtypes, Human Reprod. 13 (1998) 1578-1583.

[12] Jasko D.J., Lein D.H., Foote R.H., A comparison of two computer-automated semen analysis instrument for the evaluation of sperm motion characteristics in the stallion, J. Androl. 11 (1990) 453-459.

[13] Jasko D.J., Little T.V., Lein D.H., Foote R.H., Comparison of spermatozoal movement and semen characteristics with fertility in stallions: 64 cases (1987-1988), J. Am. Vet. Med. Assoc. 200 (1992) 979-985.

[14] Jeyendran R.S., Van der Ven H.H., Perez-Pelaez M., Crabo B.G., Zaneveld L.J., Development of an assay to assess the functional integrity of the human sperm membrane and its relationship to other semen characteristics, J. Reprod. Fertil. 70 (1984) 219-228.

[15] Leipold S.D., Graham J.K., Squires E.L., McCue P.M., Brinsko S.P., Vanderwall D.K., Effect of spermatozoal concentration and number on fertility of frozen equine semen, Theriogenology 49 (1998) 1537-1543.

[16] Massip A., Cryopreservation of embryos of farm animals, Reprod. Dom. Anim. 36 (2001) 49-55.

[17] Neild D., Chaves G., Flores M., Mora N., Beconi M., Aguero A., Hypoosmotic test in equine spermatozoa, Theriogenology 51 (1999) 721-727.

[18] Nie G.J., Development of a hypoosmotic swelling (HOS) test for stallion semen, Proc. Soc. Theriogenology, Baltimore (MA), 1998, p. 146.

[19] Pace M.M., Sullivan J.J., Effect of timing of insemination, numbers of spermatozoa and extender components on the pregnancy rate in mares inseminated with frozen stallion semen, J. Reprod. Fertil. Suppl. 23 (1975) 115-121.

[20] Padilla A.W., Foote R.H., Extender and centrifugation effects on the motility patterns of slow-cooled stallion spermatozoa, J. Anim. Sci. 69 (1991) 3308-3313.

[21] Palmer E., Factors affecting stallion semen survival and fertility, Proc. 10th Int. Congr. Anim. Reprod. 3 (1984) 377-379.

[22] Pérez L.J., Valcarcel A., de las Heras M.A., Baldassarre H., Comparative study of four techniques for evaluation of sperm quality in ovine and bovine frozen-thawed samples, Reprod. Dom. Anim. 32 (1997) 157-160.

[23] Revell S.G., Mrode R.A., An osmotic resistance test for bovine semen, Anim. Reprod. Sci. 36 (1994) 77-86.

[24] Rota A., Penzo N., Vincenti L., Mantovani R., Hypoosmotic swelling (HOS) as a screening assay for testing in vitro fertility of bovine spermatozoa, Theriogenology 53 (2000) 1415-1420. 
[25] Samper J.C., Morris C.A., Current methods for stallion semen cryopreservation: a survey, Theriogenology 49 (1998) 895-903.

[26] S.A.S. SAS/STAT User's Guide - Release 6.03, S.A.S. Institute Inc. Cary, NC, USA, 1988.

[27] Snoeck P.P.N., Henry M., Santos L.M.G., Pinheiro L.P.L., Equine semen freezing using different cryoprotective agents, Revista Brasileira de Reproducao Animal 25 (2001) 454-455.
[28] Van Der Ven H.H., Jeyendran R.S., Al-Hasani S., Perez-Pelaez M., Diedrich K., Zaneveld L.J.D., Correlation between human sperm swelling in hypoosmotic medium (Hypoosmotic Swelling Test) and in vitro fertilization, J. Androl. 7 (1986) 190-196.

[29] Watson P.F., Recent developments and concepts in the cryopreservation of spermatozoa and the assessment of their post-thawing function, Reprod. Fertil. Devel. 7 (1995) 871-891. 